\title{
Fuzzy Logic Controller for Enhancing RCS’s Lab Measurements
}

\author{
Mohamed Galal* and Jianyu Wang \\ School of Automation, Nanjing University of Science and Technology, xiaolingwei, Nanjing, Jiangsu, China \\ ${ }^{*}$ Corresponding author
}

\begin{abstract}
Precise and accurate radar cross section (RCS) measurements, especially for tracking small radar cross section targets (e.g. tracks on Fighters), or RCS reduction and radar absorbing materails evaluation for other targets of interest such as aircraft, tanks, or ships, is a must. As a consequence, the need of having more accurate evaluation for the RCS of the targets is required. This paper demonstrates the existing RCS's Lab. Then, discusses the automation of the RCS's antenna holder movements, using fuzzy logic, to have better target tracking while measurement process, in order to suppress the unwanted manmade error. Taking into account the fuzzy nature of human decision making processes and real-time properties, this paper has established different fuzzy controllers then tested and compared with a crisp decision system. The first one has been developed using an expert knowledge, the second one was learned from the recorded videos.
\end{abstract}

Keywords-RCS lab measurements enhancement; fuzzy logic control; multi-input and multi output; nonlinear system; and video guidance

\section{INTRODUCTION}

Existing outdoor RCS lab, we are specifically interested in measuring the radar-cross-section of an (aircraft fighter/civilian, Tanks, Ships, Chaffs, and Rams). If we can correctly identify and evaluate the target's RCS, more accurate information can be provided for the command and guidance systems to precisely attack the most threatening targets, or provide more help for RCS reduction scientists [1-8].

The main idea of obtaining the RCS of any target is measuring the target from each aspect angle compared to a reference target at the same field. The need to measure at each aspect angle leads to put both the transmitting, receiving antennas and the digital camera on a turn table. Vision guidance is accomplished with a CCD camera with a zoom lens. The data is collected through a commercial tracking device, communicating to the computer. In the existing lab, Vision tracking was accomplished by using a joystick to track the target maneuvers as shown in figure (1). To track while measurement process, in order to suppress the unwanted man made error, an online nonlinear controller for nonlinear tracking should be used $[9,10]$.

The control technique used for the turntable (antenna holder) used on RCS lab system must provide fast and precise performance. Poor tracking performance during measurement will result in large miss evaluation which may cause misleading results. The equations describing the RCS's lab turntable system under consideration are highly nonlinear.

Accurate nonlinear control is required in order to precisely keep the LOS between the RCS lab's antenna and the target to be measured [9]. Generally, an accurate nonlinear model of a system like that RCS's antenna holder system is difficult to find, and parameter values obtained from system identification may be only approximated values.

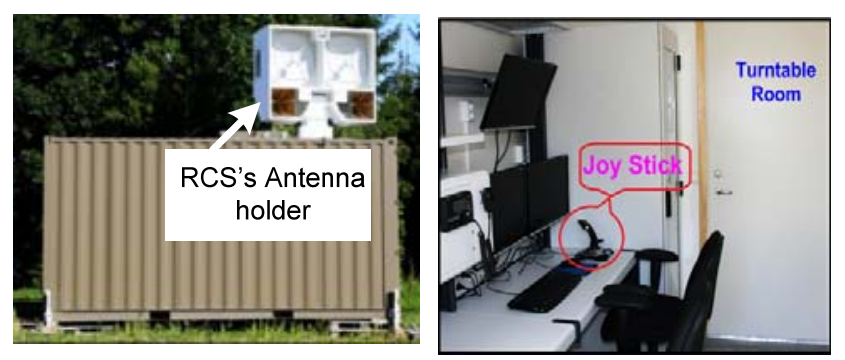

FIGURE I. OUTDOOR RCS LAB

One of the intelligent techniques, fuzzy logic developed by Zadeh [10, 11] is applied for controller design in many applications [10,11]. A fuzzy logic controller (FLC) was proved analytically to be equivalent to a nonlinear PI controller when a nonlinear defuzzification method is used [12]. Also, the results from the comparison of conventional and fuzzy logic control techniques in the form of FLC $[14,15]$ and fuzzy compensator $[16,17]$ showed that fuzzy logic can reduce the effects of nonlinearity and improve the performance of the system.

This paper uses fuzzy logic control to automate the tracking process of the RCS's Lab antenna holder. The advantages of the fuzzy logic system are that multiple types of input such as from vision and sonar sensors as well as stored map information can be used in guidance process.

Heuristic knowledge is applied to define fuzzy membership functions and rules. The membership functions and rules are then modified. The rest of this article is organized as follows. Outdoor RCS Lab theory of operation, Dynamic model of the turntable system is derived in Section two. A FLC control algorithm is proposed in Section three. Section four, The simulation model and results are illustrated. Then conclusion will be given in Sections five. 


\section{RCS LAB SYSTEM}

\section{A. Lab Description}

The main idea of obtaining the RCS of any target is measuring the target from each aspect angle compared to a reference target at the same circumstances. The need to measure at each aspect angle leads to put both the transmitting, receiving antennas and the digital camera on a turn table. Vision guidance is accomplished with a CCD camera with a zoom lens. The data is collected through a commercial tracking device, communicating to the computer. In the existing lab, Vision tracking was accomplished by using a joystick to track the target maneuvers.

Software on the radar control PC is used to setup the measurements requirements such as (Frequency, Transmitter/Receiver Polarization, Pulse Repetition Frequency, min/max range of measurements, Calibration value, etc). These settings are sent through the LAN switch to the Signal generator then to both the Transmitter/Receiver. ADC PC samples, stores, and processes both transmitted/received signals. Video Controller PC interfaces with the Joystick then controlling the antenna position by the turntable controller through the LAN switch. Figure (2) shows both processing/controlling, and turntable room. This figure shows the three computers, LAN switch, Signal generator, turntable room. Moving the antenna to track the moving target by the joystick is difficult and makes insufficient measurements. This leads to the need of automatically move the turntable to track the moving target.

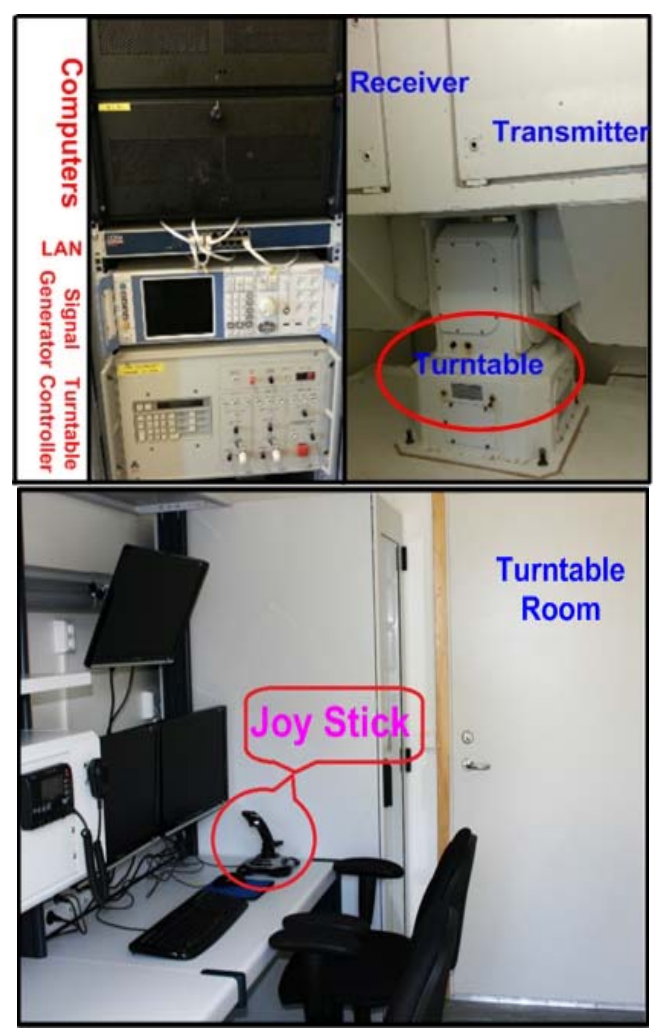

FIGURE II. PROCESSING, CONTROLLING, AND TURNTABLE ROOMS

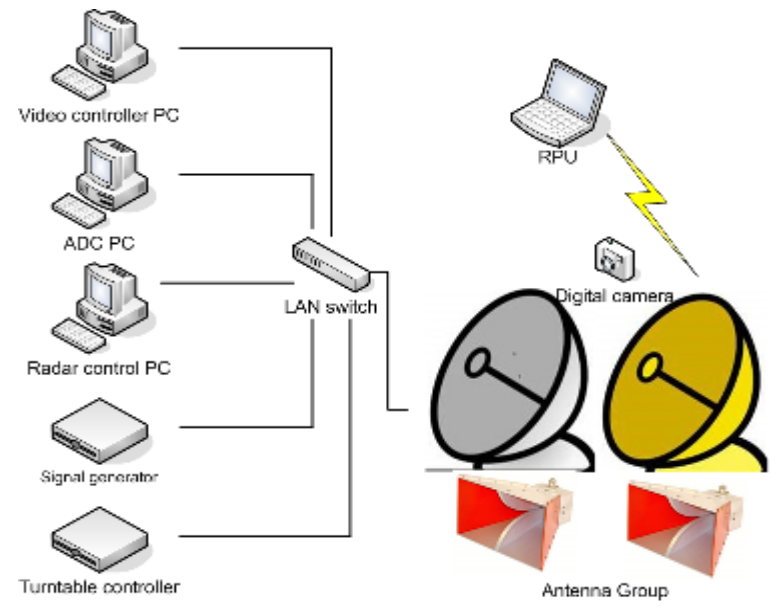

FIGURE III. RCS LAB BASIC BLOCKS

\section{B. System Model}

The dynamical analysis of the RCS's Lab antenna holder investigates a relation between the joint torques/forces applied by the actuators and the position, velocity and acceleration of the RCS turntable with respect to time. Turntable manipulators have complex non-linear dynamics that might make applying accurate and robust control difficult. Therefore, they are good examples to test performance of the controllers. The turntable system illustrated in Figure (2) consists of two revolute joints derived with two electric AC motors. According to Dinavit_Hartinberg convention the coordinate frames are considered and rearranged.

Obtaining the transformation matrices and Lagrange's equation of motion is applied to give two equations of motion, the first row describes the azimuth motion by angular coordinate $\theta_{1}$ and the second row describes the motion of the elevation by angular coordinate $\theta_{2}$.

$$
\begin{aligned}
& {\left[\left(\frac{\rho A L^{3}}{3}+m_{p} L^{2}\right) c_{2}^{2}+\left(\rho A L^{2} a+2 m_{p} L a\right) c_{2}+\left(\rho A L a^{2}+I_{R_{1}}+m_{R_{3}} a^{2}+m_{p} a^{2}+\frac{1}{2} m_{1} R_{1}^{2}\right)\right] \ddot{\theta}_{1}(1)} \\
& -\left[2\left(\frac{\rho A L^{3}}{3}+m_{p} L^{2}\right) c_{2} s_{2}+\left(\rho A L^{2} a+2 m_{p} L a\right) s_{2} \dot{\theta}_{2} \dot{\theta}_{1}=M_{1}\right. \\
& \\
& \left(\frac{\rho A L^{3}}{3}+I_{R_{2}}+m_{p} L^{2}\right) \ddot{\theta}_{2}+\left(\frac{\rho A L^{3}}{3} c_{2} s_{2}+m_{p} L^{2} c_{2} s_{2}+\frac{\rho A L^{2} a}{2} s_{2}+m_{p} L a s_{2}\right) \dot{\theta}_{1}^{2} \\
& -9.81\left(\frac{\rho A L^{2}}{2}+m_{p} L\right) c_{2}=M_{2}
\end{aligned}
$$

Where

$\theta_{1}, \theta_{2}$ angular position of motor " 1 ” and motor " 2 " respectively

$\dot{\theta}_{1}, \quad \dot{\theta}_{2} \quad$ angular speed of motor "1" and motor "2" respectively 


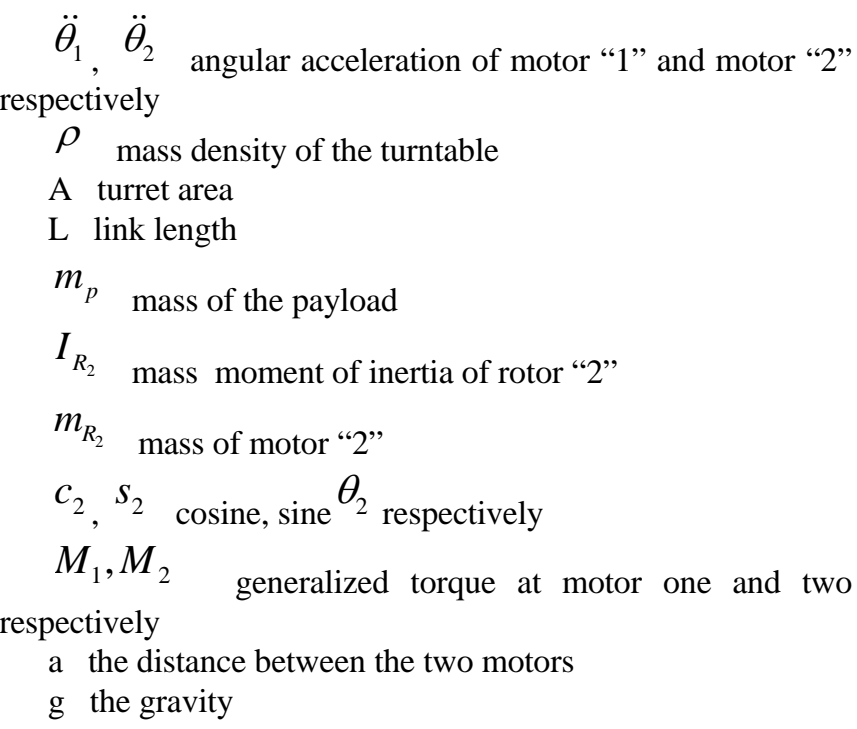

As a conclusion from these equations, this turntable is: nonlinear, multi-input-multi-output, and decoupled equations of motion, in addition to neglecting both damping and backlash effects. This conclusion leads to use a nonconventional controller.

\section{FUZZY LOGIC CONTROLlER DESIGN}

While conventional controllers depend on the accuracy of the system model and parameters, FLCs use a different approach to control the system. Fuzzy logic controller (FLC) is one of the most important applications of fuzzy logic theory. This controller can be used to control nonlinear, uncertain, and noisy systems. Instead of using system model, the operation of a FLC is based on heuristic knowledge and linguistic descriptions to the performed task. The effects from inaccurate parameters and models are reduced because a FLC does not require a system model. However, building a FLC from the ground-up may not provide good result than a conventional controller if there is not enough knowledge of the system. Therefore, in this paper, the FLC is initially constructed as a-prior knowledge in the process. The performance of the FLC is then improved by adjusting the rules and membership functions. These design procedure are described as follows.

\section{A. Procedure 1: Defining Inputs, Outputs, and Universe of Discourse}

To apply heuristic knowledge in the FLC, inputs, outputs, and universe of discourse are defined first. The inputs are the error (e) between the reference position $\left(\theta_{r}\right)$ and the actual position $\left(\theta_{a}\right)$, and the change in error $(\dot{e})$. The output is the change in armature voltage (CU). Figure (4) describes inputs, output, and FLC block diagram.

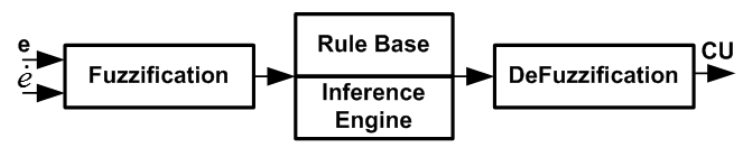

FIGURE IV. BLOCK DIAGRAM OF THE FLC

\section{B. Procedure 2: Fuzzy Membership Functions and Rules}

To perform fuzzy computation, inputs and output must be converted from numerical or crisp value into linguistic forms. The terms such as "Small” and "Big” are used to quantize inputs and output values to linguistic terms that used to represent input and output values are defined by seven fuzzy variables tabulated at table (1). Fuzzy membership functions are used as tools to convert crisp values to linguistic terms forms.

TABLE I. FUZZY LINGUISTIC TERMS

\begin{tabular}{|l|l|}
\hline Term & Definition \\
\hline NB & Negative big \\
\hline NM & Negative medium \\
\hline NS & Negative small \\
\hline ZE & zero \\
\hline PS & Positive small \\
\hline PM & Positive medium \\
\hline PB & Positive big \\
\hline
\end{tabular}

A fuzzy membership function can contain several fuzzy sets depending on how many linguistic terms. In this paper, seven fuzzy sets are obtained by applying the seven linguistic terms. The number for indicating how much a crisp value can be a member in each fuzzy set is called a degree of membership. One crisp value can be converted to be partly in many fuzzy sets, but the membership degree in each fuzzy set may be different. In order to define fuzzy membership function, designers can choose many different shapes based on their preference or experience. The popular shapes are triangular and trapezoidal because these shapes are easy to represent designer's ideas and require low computation time.

For performing fine-tuning to improve the efficient of the controller, the adjacent of each fuzzy set value should overlap by about 25\% [19]. The membership functions are illustrated in Figure (4). In equation (1), we see that the equation of motion are in a nonlinear form. We ought to use FLC to overcome this nonlinearity of the equation of motion of the system to be controlled. Instead of using mathematical formulas, a FLC uses fuzzy rules to make a decision and generate the control effort [20]. The rules are in the form of IF-THEN statements. For example, IF the error (e) is equal to Positive Big (PB) and the change in error $(\dot{e})$ is equal to Positive

Medium (PM) THEN the change in control signal (CU) is equal to Negative Medium (NM). The matters in defining rules are how many rules should be used and how to determine the relation in IF-THEN statements. Actually, the solutions are based on the experience of a designer or the previous knowledge of the system. The critical point is if there is not sufficient knowledge applied in the design, the result could be drastically bad. The rules are constructed as shown in Table (2).

To send out the armature voltage output, the output in the form of fuzzy sets must be converted to a crisp value. This process is called defuzzification. In current article, the center of gravity method is chosen. Figure (3) illustrates the initial membership functions used by the initial FLC. Seven triangular shape membership functions are used for each of the inputs and 
output. The efficiency can be improved by adjusting membership functions and rules in procedure.

\section{Procedure 3: Adjusting Membership Functions \& Rules}

In order to improve FLC performance, rules and member-ship functions are adjusted by making the area of member-ship functions near ZE region narrower to produce finer control resolution. On the other hands, making the area far from ZE region wider gives faster control response.

Also, the performance can be improved by changing the severity of the rules [21]. After adjusting the rules and membership functions, the final rules and membership functions are obtained as shown in, Table 3 and Figure (5) respectively.

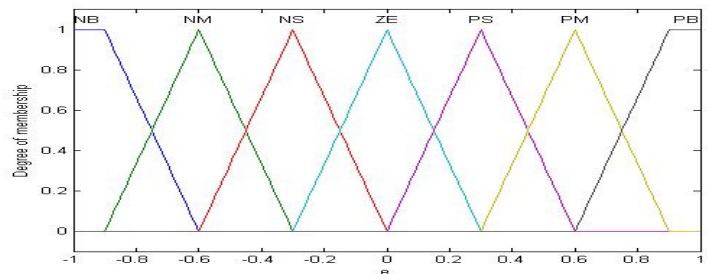

FIGURE V. MEMBERSHIP FUNCTIONS

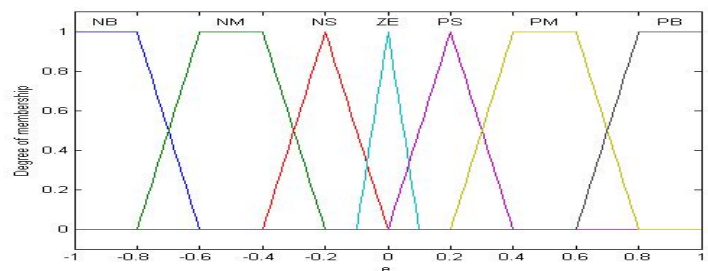

FIGURE VI. MODIFIED MEMBERSHIP FUNCTIONS

TABLE II. INITIAL RULES

\begin{tabular}{|c|c|c|c|c|c|c|c|}
\hline a & NB & NM & NS & ZE & PS & PM & PB \\
\hline NB & NB & NB & NB & NB & NM & NS & ZE \\
\hline NM & NB & NB & NB & NM & NS & ZE & PS \\
\hline NS & NB & NB & NM & NS & ZE & PS & PM \\
\hline ZE & NB & NM & NS & ZE & PS & PM & PB \\
\hline PS & NM & NS & ZE & PS & PM & PB & PB \\
\hline PM & NS & ZE & PS & PM & PB & PB & PB \\
\hline PB & ZE & PS & PM & PB & PB & PB & PB \\
\hline
\end{tabular}

TABLE III. MODIFIED RULES

\begin{tabular}{|c|c|c|c|c|c|c|c|}
\hline $\mathbf{a}$ & NB & NM & NS & ZE & PS & PM & PB \\
\hline NB & NB & NB & NB & NB & ZE & PM & PM \\
\hline NM & NB & NB & NB & NM & PS & PM & PB \\
\hline NS & NB & NB & NM & NS & PM & PM & PB \\
\hline ZE & NB & NM & NS & ZE & PB & PB & PB \\
\hline PS & NM & NS & ZE & PS & PM & PB & PB \\
\hline PM & NS & ZE & PS & PM & PB & PB & PB \\
\hline PB & ZE & PS & PM & PB & PB & PB & PB \\
\hline
\end{tabular}

\section{Simulation Results}

Matlab/Simulink software is used to: simulate the turntable's equations of motion, and to make a good comparison between performances of FLC before (initial FLC) and after (modified FLC) adjusting the fuzzy membership functions and rules. Step and half wave sinus signals were applied to the system as a control inputs. Both the controllers' step and sinus responses of the system were given in Figures (6-9) respectively. For this work, rising times and overshot according to the modified FLC are better than in the original one which its performance is out of the control aim. These results illustrate good performance of the system under control of the modified FLC than that under control of the initial FLC.

\section{CONCLUSIONS}

A detailed RCS Lab construction was illustrated and discussed. Then the importance of automatically tracking targets that improves the target's RCS measurements was mentioned. After driving the equations of motion of the turntable (RCS's antenna holder), we found that it is a multi-input/output system. In this work, the application of fuzzy logic in outdoor RCS Lab based on the expert knowledge is discussed, analyzed and simulated. Two different fuzzy systems have been tested and compared with a crisp decision system. The first one has been developed using an expert knowledge, and as a refinement, the second one was modified according to the recorded videos. Results obtained show better capabilities for the system to improve tracking, then enhancing the real target RCS measurements.

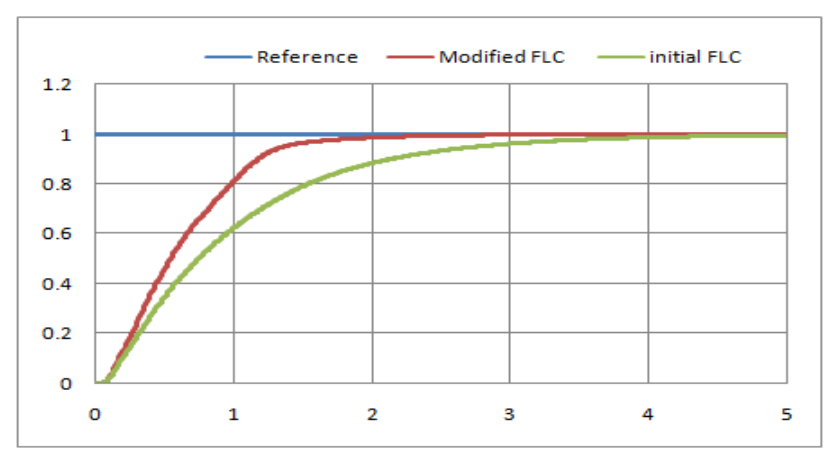

FIGURE VII. STEP RESPONSE IN AZIMUTH PLAN

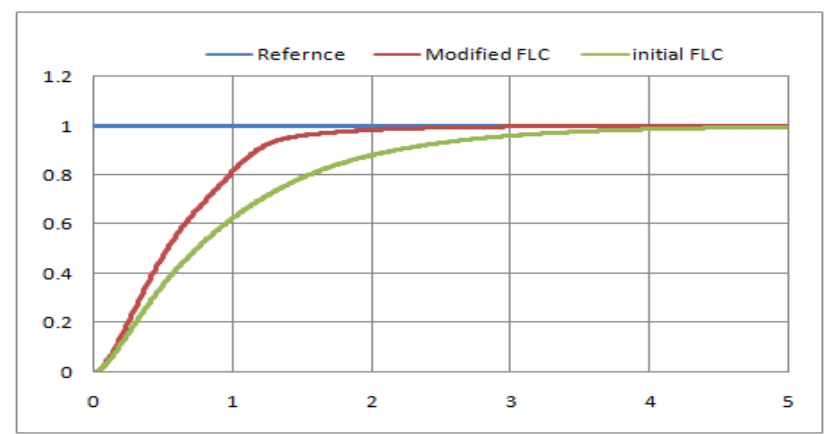

FIGURE VIII. STEP RESPONSE IN ELEVATION PLAN 


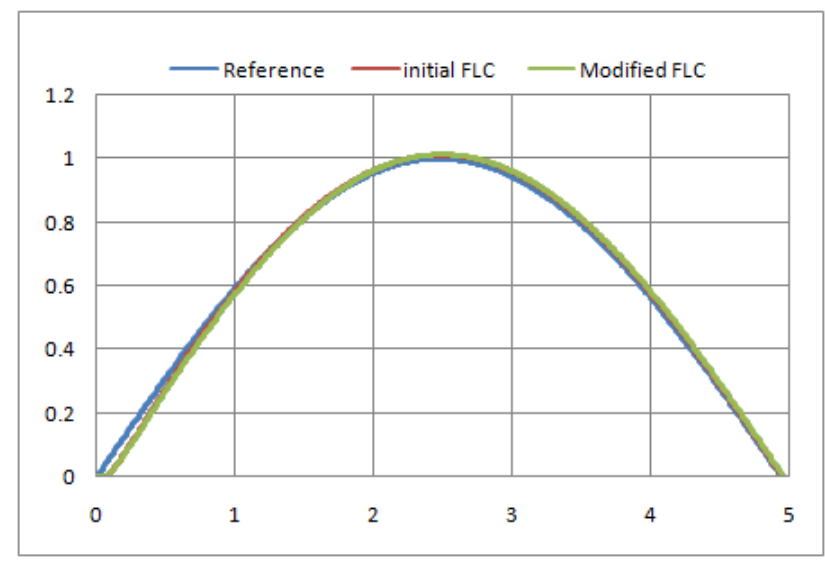

FIGURE IX. SINUS RESPONSE IN AZIMUTH

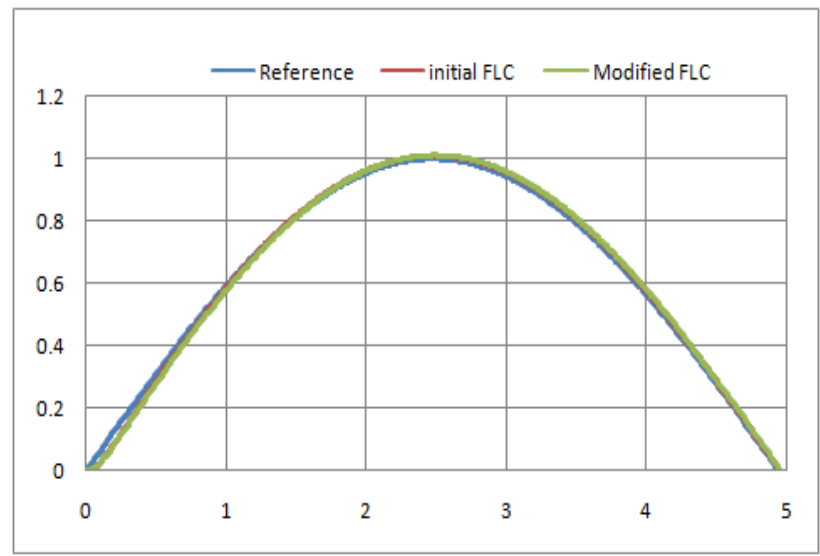

FIGURE X. SINUS RESPONSE IN ELEVATION

\section{REFERENCES}

[1] S. Challa, G.W. Pulford, Joint target tracking and classification using radar and ESM sensors, IEEE Trans. Aerosp. Electron. Syst. 37 (2001) 1039-1055.

[2] Y. Boers, H. Driessen, Integrated tracking and classification: an application of hybrid state estimation, Proc. SPIE Signal Data Process. Small Targets 4473 (2001) 198-209.

[3] N. Gordon, S. Maskell, T. Kirubarajan, Efficient particle filters for joint tracking and classification, Proc. SPIE Signal Data Process. Small Targets 4728 (2002) 439-449.

[4] M.I. Miller, A. Srivastava, U. Grenander, Conditional-mean estimation via jump-diffusion processes in multiple target tracking/recognition, IEEE Trans. Signal Process. 43 (1995) 2678-2690.

[5] D. Lanterman, Tracking and recognition of airborne targets via commercial television and FM radio signals, Proc. SPIE Acquis., Track., Pointing 3692 (1999) 189-198.

[6] S. Herman, P. Moulin, A particle filtering approach to FM-band passive radar tracking and automatic target recognition, in: Proceedings of the IEEE Aerospace Conference, vol. 4, pp. 1789-1808, 2002.

[7] Shawn Michael Herman, A particle filtering approach to joint passive radar tracking and target classification (Ph.D. dissertation), US: Graduate College of the University of Illinois, 2002.

[8] Hong Jiang, Long Xu, Kun Zhan, Joint tracking and classification based on aerodynamic model and radar cross section, Pattern Recognition, Volume 47, Issue 9, September 2014, Pages 3096-3105.

[9] Meng Joo Er, and Ya Lei Sun, Hybrid Fuzzy Proportional-Integral Plus Conventional Derivative Control of Linear and Nonlinear Systems, IEEE
TRANSACTIONS ON INDUSTRIAL ELECTRONICS, VOL. 48, NO. 6 , DECEMBER 2001.

[10] Shao, H.-F, Hu, B.-G., Zhu, Z.-L., A case study of one-to-two mapping fuzzy PD controllers on inverted pendulum, Fuzzy Systems Conference Proceedings, 1999.

[11] Jung-Wook Park, Harley, R.G., Venayagamoorthy, G.K., New external neuro-controller for series capacitive reactance compensator in a power network, Power Systems, IEEE Transactions on (Volume:19, Issue: 3 ) 2004.

[12] Dawood Amoozegar, DSTATCOM modeling for voltage stability with fuzzy logic PI current controller, International Journal of Electrical Power \& Energy Systems, Volume 76, March 2016, Pages 129-135.

[13] Basil Mohammed Al-Hadithi, Antonio Javier Barragán, José Manuel Andújar, Agustín Jiménez; Chattering-free fuzzy variable structure control for multivariable nonlinear systems Applied Soft Computing, Volume 39, February 2016, Pages 165-187.

[14] Zha Xu, Hu Yu nan, Cui Ping yuan, A study of terrain following controller based on back stepping and variable structure control, Intelligent Control and Automation, 2004. WCICA 2004. Fifth World Congress on Volume: 6.

[15] Essam Natsheh, and Khalid A. Buragga, Comparison between Conventional and Fuzzy Logic PID Controllers for Controlling DC Motors; IJCSI International Journal of Computer Science Issues, Vol. 7, Issue 5, September 2010.

[16] Kacprzyk, J., Zadrozny, Computing With Words Is an Implementable Paradigm: Fuzzy Queries, Linguistic Data Summaries, and Natural-Language Generation, Fuzzy Systems, IEEE Transactions on Volume: 18 , Issue: 3, 2010.

[17] Zarandi, M.H.F., Amir kabir, Sedehizadeh, , A hybrid approach to develop an interval type-2 fuzzy logic system, Fuzzy Information Processing Society (NAFIPS), 2012 Annual Meeting of the North American.

[18] Hagras, H., Developing a type-2 FLC through embedded type-1 FLCs, Fuzzy Systems, 2008. (IEEE World Congress on Computational Intelligence).

[19] Feng Xue, Sanderson, A.C., Bonissone, P.P., Graves, R.J., Fuzzy Logic Controlled Multi-Objective Differential Evolution, Fuzzy Systems, 2005. The 14th IEEE International Conference.

[20] Viswanathan, K. ; Oruganti, R. ; Nonlinear function controller: a simple alternative to fuzzy logic controller for a power electronic converter, Industrial Electronics, IEEE Transactions on Volume:52 , Issue: 5, 2005.

[21] M. Galal, N. G. Mikhail, G. Elnashar, Fuzzy logic Controller Design for gun-turret system, 13th International Conference on AEROSPACE SCIENCES \& AVIATION TECHNOLOGY, ASAT- 13, May 26 - 28, 2009. 